\title{
Fiscalización y cumplimiento ambiental en Chile: principales avances, desafíos y tareas pendientes
}

Kay Bergamini. Pontificia Universidad Católica de Chile, Santiago, Chile. Cristian Pérez. Universidad de Chile, Santiago, Chile.

RESUMEN | El año 2010 se promulgó la Ley 20.417, que creó la Superintendencia del Medio Ambiente (sMA), la cual entró en plenas funciones el 28 de diciembre de 2012. Los principales elementos de diseño de esta nueva institución se basaron en los resultados de las Evaluaciones del Desempeño Ambiental llevadas a cabo por la Organización para la Cooperación y el Desarrollo Económicos (OCDE) en 2005, los que fueron indicados en el discurso de la Presidenta de la República el año 2008, al momento del ingreso del proyecto de ley propiamente tal. Si bien resultan evidentes los adelantos en materia de fiscalización y cumplimiento ambiental respecto del modelo de coordinación desarrollado e implementado desde 1994 a 2012, es necesario avanzar en incrementar competencias en el ámbito regional. El propósito de tales incrementos es limitar los conflictos de interés y superar los desbalances territoriales mostrados en términos de fiscalización, o las situaciones en que se vulnera la justicia ambiental, asociadas a la interpretación de los Planes de Descontaminación en distintas áreas urbanas del país. Junto con ello, se debe implementar mejoras legales sustanciales para que operen eficazmente los mecanismos de incentivo al cumplimiento de Planes de Reparación y Autodenuncia, entre otros aspectos.

palabras clave | Superintendencia del Medio Ambiente (Chile), fiscalización ambiental, cumplimiento ambiental, institucionalidad ambiental pública.

SUMMARY | On 2010 it was enacted the Law 24.417 that created the Superintendence of the Environment (Superintendencia del Medio Ambiente, SMA), the same that was fully operational on December 28, 2012. The main design elements for this new agency were based on the outcomes of the Environmental Performance Review carried out by the Organisation for Economic Co-operation and Development (OECD) on 2005, included at the President's speech of 2008 at the moment of submitting the final bill for consideration. While there are evident advances on environmental enforcement matters-compared to the coordination model implemented from 1994 to 2012- it is necessary to move forward towards increasing competences on the regional realm. This development's primary aims would be both to reduce conflicts of interest and to overcome the territorial imbalances showed in terms of the Decontaminating Plans in different urban areas of the country. At the same time, substantial legal improvements must be implemented to advance mechanisms for the compliance of the Restoration Plans and Self-Denounces, among other matters.

KEYWORDS | Superintendence of the Environment (Chile), environmental enforcement, environmental compliance, public institutional framework. 


\section{Antecedentes históricos}

En 1994, el Estado de Chile promulgó la Ley 19.300 sobre Bases Generales del Medio Ambiente. La estructura institucional ambiental propuesta en dicha ley fue construida siguiendo el modelo de la "propuesta de Ley básica de protección ambiental y promoción del desarrollo sostenible" de 1993, elaborada para América Latina por el Programa de las Naciones Unidas para el Medio Ambiente (PNUMA), que formulaba los criterios y normas básicas destinadas a regular la gestión ambiental del Estado y las acciones de los habitantes, a fin de alcanzar los objetivos del desarrollo sostenible del país (Olivares, 2010).

De la misma forma, proponía el desarrollo de un sistema de evaluación ambiental, organizado en torno a dos niveles de control administrativo: la licencia ambiental y el estudio de impacto ambiental; promovía la educación y capacitación ambiental; incentivaba la elaboración de un régimen de beneficios e incentivos económicos, destinado a las empresas que colaborasen con el desarrollo sostenible; y planteaba la creación de una Comisión Nacional del Medio Ambiente (Conama), como órgano coordinador de las políticas ambientales. Por último, impulsaba la gestión de los recursos naturales renovables de conformidad a las exigencias del desarrollo sostenible. Sin embargo, pese a que la propuesta del pNuma fue implementada en gran parte por la Ley 19.300, inspirando el desarrollo del sistema institucional ambiental nacional, aunque de forma atenuada, no acogió la sugerencia de otorgar el rango de ministro al presidente de la Conama, como tampoco incorporó el delito ambiental dentro de la legislación penal, entre otros puntos esenciales de la proposición (Olivares, 2010).

Según señala Cordero (1996), al radicar la institucionalidad ambiental en una comisión de carácter intersectorial como era Conama, la Ley 19.300 se apartó de nuestra realidad organizacional e hizo suya una regulación normativa ajena a nuestro modo de ser y entender la Administración Pública, y coincidente con el proyecto de la ley marco del medioambiente para Centroamérica y Panamá.

En relación con la fiscalización ambiental, la estructura del modelo se basó en mantener las competencias respectivas en los ministerios y organismos sectoriales del Estado a los cuales hasta ese momento estaban asignadas. Debido a la aplicación de este modelo, la Conama se enfrentó a un escenario en que coexistían diferentes metodologías de fiscalización ambiental, y sin control sobre los diferentes órganos del Estado involucrados en ellas. Lo anterior, más la dispersión de regulaciones ambientales en diversos instrumentos, funciones sobrepuestas de organismos del Estado y falta de procedimientos de información entre sus mismos órganos, fueron factores que obstaculizaron la fiscalización y cumplimiento de la legislación ambiental en Chile.

Las deficiencias antes señaladas fueron factores que justificaron la revisión del modelo y su subsecuente reforma, lo que fue reforzado por varios reportes y evaluaciones a nivel nacional e internacional. De la mayor importancia fue la publicación del informe Evaluaciones del desempeño ambiental, generado por la Organización para la Cooperación y el Desarrollo Económicos (OCDE) y la Comisión Económica para América Latina y el Caribe (CEPAL) el año 2005. En dicho informe, enfocado 
en la situación ambiental de Chile y desarrollado en el marco de la intención del país de ser parte de dicha Organización, fue relevado el hecho de que una política de fiscalización ambiental basada en la coordinación de varios órganos de fiscalización sectorial, no es la forma más efectiva de organización institucional para asegurar el cumplimiento con la legislación ambiental (oCDE \& CEPAL, 2005).

En términos prácticos, las principales dificultades asociadas a dicho modelo se relacionaban con diversos factores: el hecho de que las capacidades ambientales estuvieran localizadas en agencias sectoriales, sin una mirada puramente ambiental; la duplicidad de funciones, con el consecuente incremento de costos públicos; multas bajas; ausencia de programas y metodologías para desarrollar actividades de fiscalización ambiental apropiadas; e incentivos puestos en las sanciones en vez del cumplimiento ambiental (Monckeberg, Bergamini \& Pérez, 2015).

Así, como consecuencia de tales dificultades, la revisión llevada a cabo por la ocDE y CEPAL recomendó al Estado de Chile:

- desarrollar y fortalecer las instituciones ambientales en los ámbitos nacional y regional; $y$

- desarrollar y fortalecer aún más los marcos normativos para mejorar la salud ambiental y cumplir los compromisos internacionales de Chile; examinar formas de fortalecer la capacidad de cumplimiento y fiscalización, incluso mediante reformas institucionales, como por ejemplo el establecimiento de un órgano de inspección ambiental.

En el contexto antes descrito y en el marco de los compromisos adquiridos por el país, el año 2010 se promulgó la Ley 20.417 que creó el Ministerio del Medio Ambiente (MMA), el Servicio de Evaluación Ambiental (sEa) y la Superintendencia del Medio Ambiente (sma). Esta última agencia, en particular, correspondía a la recomendación de establecer un órgano de inspección ambiental específico.

\section{Elementos de diseño del nuevo modelo ambiental en Chile}

Analizando más profundamente el documento elaborado por la OCDE y CEPAL (2005) y el mensaje presidencial, enviado el día 5 de junio de 2008, para iniciar la tramitación de la Ley 20.417 (Biblioteca del Congreso Nacional [BCN], 2010), en relación con la fiscalización, sanción y cumplimiento ambiental en Chile, los principales elementos de diseńo que se tienen en cuenta se detallan en la Tabla 1.

No obstante lo visto en la Tabla 1, llamativo fue que una de las principales sugerencias de la OCDE y CEPAL (2005) no haya sido tomada en cuenta, y que dice relación con que "el papel fiscalizador asignado a las administraciones sectoriales debería incrementarse en el ámbito regional, donde los costos de transacción y los conflictos de interés son bajos en comparación con los niveles central y local" (p. 145, destacados en el original).

A continuación se realiza un análisis crítico desde la perspectiva de identificar los principales avances, desafíos y tareas pendientes, incluyéndose además una mirada desde el contexto territorial en cuanto a la fiscalización, sanción y otros aspectos que puedan ser relevantes. 
TABLA I | Elementos de diseńo del nuevo modelo ambiental en Chile

\begin{tabular}{|c|c|c|}
\hline FISCALIZACIÓN & SANCIÓN & OTROS ELEMENTOS \\
\hline $\begin{array}{l}\text { Fiscalización múltiple. A través } \\
\text { de la propia sMA, de los órganos } \\
\text { sectoriales e incluso por parte de } \\
\text { terceros debidamente certifica- } \\
\text { dos, para limitar el denominado } \\
\text { "Predominio del Enfoque } \\
\text { Sectorial". } \\
\text { Establecimiento de Progra- } \\
\text { mas Anuales de Fiscalización, } \\
\text { donde se coordine el accionar del } \\
\text { Estado, para así evitar el escaso } \\
\text { control de la fiscalización y cum- } \\
\text { plimiento de órganos sectoriales, } \\
\text { pero también la acción dispersa } \\
\text { e inorgánica, lo cual genera } \\
\text { muchos costos. } \\
\text { Implementación de un sistema } \\
\text { de Evaluación y Certificación de } \\
\text { Conformidad, con regulación de } \\
\text { la sma y para ser ejecutada por } \\
\text { sujetos (privados) certificados. } \\
\text { Otorgamiento de amplias atri- } \\
\text { buciones, que permiten registro, } \\
\text { requerimientos de información y } \\
\text { citaciones de personas. }\end{array}$ & $\begin{array}{l}\text { Establecimiento de la Autode- } \\
\text { nuncia, Programa de Cumpli- } \\
\text { miento y Planes de Reparación, } \\
\text { como mecanismos de incentivo } \\
\text { que permiten corregir infrac- } \\
\text { ciones y disminuir monto de } \\
\text { multas. } \\
\text { Sanciones disuasivas, incluyendo } \\
\text { un registro público de sanciones, } \\
\text { con montos de dinero consi- } \\
\text { derables e incluso la clausura o } \\
\text { la suspensión de sus permisos } \\
\text { ambientales y publicidad por } \\
\text { estos actos. } \\
\text { Establecimiento de un único } \\
\text { procedimiento sancionador, de } \\
\text { forma de evitar duplicidades en } \\
\text { servicios públicos y en sanciones } \\
\text { por el mismo hecho. } \\
\text { Responsabilidad en sanciones, } \\
\text { estableciendo que los represen- } \\
\text { tantes de las empresas o titulares } \\
\text { son también responsables en el } \\
\text { caso de la multas. }\end{array}$ & $\begin{array}{l}\text { Implementación de la Denuncia } \\
\text { Ciudadana en temas ambientales, } \\
\text { que obliga a la sma a investigar y } \\
\text { al denunciante a ser parte intere- } \\
\text { sada del procedimiento. } \\
\text { Facultad para establecer medidas } \\
\text { provisionales, en caso de de- } \\
\text { tectarse situaciones de riesgo o } \\
\text { contingencia ambiental. }\end{array}$ \\
\hline
\end{tabular}

FUENTE ELABORACIÓN PROPIA A PARTIR DE OCDE Y CEPAL (2005) Y BCN (20IO)

\section{Fiscalización}

Si bien puede considerarse como avance en materia de fiscalización el establecimiento de programas y subprogramas de fiscalización, debido a que ha conseguido ordenarse la ejecución de las actividades de inspección ambiental, muestreos, análisis y examen de información por parte de los organismos públicos que cuenten con convenio de encomendación de acciones, los datos reales han demostrado presentar una notoria inequidad regional respecto de los resultados efectivos. Tal como se observa en la Tabla 2, el total de actividades de inspección ambiental, que se refiere a la ejecución de labores en terreno, presenta diferencias de hasta 127 veces entre la región que más ejecutó (Metropolitana de Santiago) con la que menos lo hizo (Tarapacá). Si el total de actividades es normalizado por el total de población por región estimado a 2014, existe un diferencial de 38 veces entre la región que mejor indicador tiene (O’Higgins) y la peor aspectada (Biobío). 
CUADro 2 Actividades de inspección ambiental ejecutadas por la SMA u organismos subprogramados el año 2014. Destacadas en gris oscuro aquellas regiones que presentan el menor número de actividades de fiscalización por habitante, y en gris claro aquellas que presentan el mayor número por habitante

\begin{tabular}{|c|c|c|c|}
\hline REGIÓN & $\begin{array}{l}\text { ACTIVIDADES } \\
\text { DE INSPECCIÓN } \\
\text { AMBIENTAL } \\
20 I 4\end{array}$ & $\begin{array}{c}\text { POBLACIÓN } \\
2014\end{array}$ & $\begin{array}{c}\text { TASA } \\
\text { (POBLACIÓN/ } \\
\text { ACTIVIDADES } \\
\text { DE INSPECCIÓN } \\
\text { AMBIENTAL) }\end{array}$ \\
\hline Arica y Parinacota & 26 & 235.081 & 9.042 \\
\hline Tarapacá & 23 & 328.782 & 14.295 \\
\hline Antofagasta & 49 & 613.328 & 12.517 \\
\hline Atacama & 28 & 308.247 & 11.009 \\
\hline Coquimbo & 27 & 759.228 & 28.120 \\
\hline Valparaíso & 36 & 1.808 .300 & 50.231 \\
\hline Metropolitana de Santiago & 2.938 & 7.228 .581 & 2.460 \\
\hline Libertador Gral. Bernardo O’Higgins & 676 & 910.577 & 1.347 \\
\hline Maule & 49 & 1.035 .593 & 21.135 \\
\hline Biobío & 41 & 2.100 .494 & 51.232 \\
\hline La Araucanía & 210 & 983.499 & 4.683 \\
\hline Los Ríos & 30 & 401.548 & 13.385 \\
\hline Los Lagos & 60 & 834.714 & 13.912 \\
\hline Aisén del Gral. Carlos Ibáñez del Campo & 30 & 107.334 & 3.578 \\
\hline Magallanes y de la Antártica Chilena & 31 & 163.748 & 5.282 \\
\hline Interregional* & 18 & - & - \\
\hline Total & 4.272 & 17.819 .054 & 4.171 \\
\hline
\end{tabular}

* En el caso de Inspecciones Ambientales de Resoluciones de Calificación Ambiental, se PUEDEN EJECUTAR ACTIVIDADES QUE INVOLUCREN MÁS DE UNA REGIÓN Y CUYA CUENTA NO ES POSIBLE ASIGNAR A UNA REGIÓN ESPECÍFICA.

FUENTE ELABORACIÓN PROPIA A PARTIR DE CUENTA PÚBLICA SUPERINTENDENCIA DEL MEDIO AMbiente (SMA, 20I5) E InSTituto NACional De ESTADísticas (INe, 2015)

Con respecto a temas de fiscalización ambiental, es importante destacar que el enfoque sectorial de los servicios públicos, identificado como uno de los problemas en materia ambiental por la oCDE y CEPAL (2005), en vez de subsanarse con la nueva institucionalidad, paradójicamente se ha visto incrementado. Dentro de las situaciones que explican este fenómeno se pueden indicar las siguientes:

a. Dado que la fiscalización ambiental ya no está dentro de sus funciones primarias debido a la pérdida de atribuciones ambientales por parte de los organismos públicos, las instituciones han ido orientando o modificando las actividades de los fiscalizadores ambientales hacia otras materias sectoriales de su competencia (por ejemplo, en el caso del Ministerio de Salud, las actividades subprogramadas 
disminuyeron de 266, equivalentes al 66\% del programa el año 2013 [sma, 2014a], a 105, equivalentes a 35\% del programa el ańo 2015 [sMA, 2014b]).

b. Lo anterior se ha visto reforzado por la pérdida de potestad sancionatoria, debido a que el incentivo está en fiscalizar aquellos aspectos donde sí tengan competencia, de forma que el propio Servicio pueda sancionar y de cierta forma llevarse el "crédito político" por dicha situación.

c. La incorporación de Indicadores de Desempeño en los organismos sectoriales, en los términos que estaba previsto en los artículos 17 y 23 de la Ley Orgánica de la Superintendencia del Medio Ambiente (LO-SMA), no ha ocurrido de acuerdo con lo normado, tanto porque no es prioridad para el servicio sectorial, por lo indicado previamente, como por el hecho de que tampoco se encuentra dentro de las prioridades de la Dirección de Presupuesto (Dipres), dejándose en indicadores de menor importancia, que impiden un adecuado compromiso por parte de dichos organismos.

d. Finalmente, si bien se establecen presupuestos sectoriales para fiscalización ambiental, la ausencia de glosas específicas en la Ley de Presupuestos, que obliguen a destinar montos de dinero para fines específicos en la materia, provoca que el financiamiento se confunda con otras prioridades de los servicios públicos.

También se han presentado, desde la perspectiva práctica, algunas situaciones de fiscalización inorgánica, siendo complejo para la Superintendencia resolver este problema. Ejemplos donde se ha verificado esta situación incluyen:

- Fiscalización sanitaria del Servicio Nacional de Pesca (Sernapesca). Esta es derivada como denuncia, en casos de que centros de cultivo de salmónidos tengan Resolución de Calificación Ambiental (RCA), provocando el ingreso de un número importante de fiscalizaciones que no están contempladas en los programas de fiscalización y, por lo tanto, en el tiempo de los profesionales para realizar informes y posteriormente procedimientos sancionatorios. Esta misma situación se repite con otros organismos, tales como Servicios de Salud, Servicio Agrícola y Ganadero (sAG), Superintendencia de Servicios Sanitarios (sISs), entre otros.

- Planes de Descontaminación, por ejemplo de Temuco y Padre Las Casas. Durante episodios críticos, la Secretaría Regional Ministerial (Seremi) de Salud decreta Alerta Sanitaria, un mecanismo de carácter sectorial, por lo tanto, al margen de la supervisión de la SMA, fiscalizando un número indeterminado de actividades y sancionando al margen del Plan. En este mismo sentido, la estrategia de implementación de Planes de Prevención y Descontaminación (MMA, 2014) es "institucionalizar" la aplicación de Alertas Sanitarias en el resto de las ciudades afectas a estos instrumentos, lo que es un resquicio legal e inhabilita la aplicación de la Ley 20.417 y, por ende, el actuar de la sMA. 


\section{Sanción y denuncias}

En primer término, se puede evaluar como un avance la implementación de mecanismos de incentivo al cumplimiento. En ese contexto, la presentación de los Programas de Cumplimiento, con 73 casos (32 en 2013 y 41 en 2014) ha sido el más utilizada por los titulares, en comparación con las Autodenuncias (6 casos en 2013 y 2014) y los Planes de Reparación (ningún caso en 2013 y 2014). Este hecho se da por los beneficios que han encontrado los infractores en dicho instrumento, en términos que da por concluido el procedimiento sancionatorio, y de esta forma inhibe tanto la multa como el ingreso al registro público de sanciones, con lo que se evitan largas tramitaciones e impactos al regulado. No obstante lo anterior, estos mismos instrumentos son los que presentan una mayor problemática, específicamente en los siguientes aspectos:

a. Con respecto de los Programas de Cumplimiento, donde este instrumento presenta dificultades en relación con su validación por parte de la ciudadanía, ya que resulta complejo de comprender, sobre todo el hecho de que empresas que no hayan cumplido con sus obligaciones ambientales queden exentas de sanción. Es necesario, así, mejorar los mecanismos de información, o establecer limitaciones respecto de su uso.

b. Mayor gravedad reviste el caso de los Planes de Reparación, que debe ser abordado desde la perspectiva de la gestión y definición de roles. A la fecha no existen presentaciones sobre tales planes, los que deben ser informados por el Servicio de Evaluación Ambiental, de manera que se permita llevar a cabo un seguimiento efectivo respecto de la reparación de un dańo ambiental cometido. Esto debido a que, en la actualidad, se están tramitando causas por dańo ambiental en el Tribunal Ambiental, el cual -en casos como Colhue (Segundo Tribunal Ambiental, 2015)- ha fijado un acuerdo para reparar el dańo ambiental sin que sea visto por la sma. Tal dańo no fue evaluado por el Servicio de Evaluación Ambiental y, más importante aún, el seguimiento del cumplimiento del acuerdo se ha producido al margen de la autoridad administrativa, puesto que los informes de monitoreo son reportados directamente al Tribunal Ambiental, con lo que en la práctica ocurre un conflicto de roles.

c. Por último deben considerarse las autodenuncias, instrumento que no han gozado del éxito esperado, con solo seis ocasiones en que ha sido utilizado en dos años. Ello puede deberse al elevado estándar legal y técnico establecido en la normativa, que provoca que su utilización sea acotada a un universo reducido de infracciones. Otro desincentivo a su utilización radica en que el ingreso mediante Programas de Cumplimiento tiene el efecto de limitar la sanción en caso de su falta, por lo que no es necesario exponerse a este trámite. El hecho de que sea aplicable por titular y no por proyecto, hace de él un instrumento atractivo para aquellos titulares con un reducido número de RCA, en comparación con titulares con un número elevado de RCA. 
Otro de los aspectos críticos a la fecha ha sido la baja capacidad de generar procedimientos sancionatorios por parte de la Superintendencia, debido más que a temas técnico-jurídicos, al volumen de trabajo que ha significado para la institución. Si se toma de base la información de la cuenta pública de 2013 (sMA 2014c) solo para RCA, se tiene que el $65 \%$ de los procedimientos de fiscalización (283 casos) fue derivado para el inicio de procedimientos sancionatorios, iniciándose solo 70, quedando más de 210 casos sin ser vistos por la SMA. En ese contexto y si bien existe un plan para aumentar la dotación de fiscales instructores de procedimientos sancionatorios en el año 2015, se hace necesario perfeccionamientos en la ley que permitan establecer trámites más expeditos o estandarizados para ciertas infracciones tipo (por ejemplo, aquellas derivadas de Normas de Emisión) o establecer una capacidad sancionadora regional, dejando el nivel central como una instancia de apelación interna del organismo.

Finalmente, se han detectado algunos temas que no fueron tratados adecuadamente durante la tramitación de la ley, como es la sanción asociada a planes de descontaminación o algunas normas de emisión que pueden afectar a ciudadanos comunes, donde las multas mínimas parten en 1 Unidad Tributaria Anual (UTA), (aprox. Us\$1.000), lo que se puede considerar alto para personas naturales. Aún más grave que lo anterior resulta el hecho de que en el caso del Plan de la Región Metropolitana de Santiago no existe una sanción por la SMA y cada organismo sectorial sanciona de manera independiente, lo que contradice el espíritu de la Ley 20.417, generando distorsiones con características de Justicia Ambiental (Hervé, 2010).

En relación con las denuncias, existe una muy baja capacidad de atenderlas adecuadamente, debido a que la realidad superó las expectativas. De acuerdo con información aportada por la SMA (2014c y 2015), hubo más de 1.600 denuncias el año 2013, mientras el año 2014 se llegó a 1.499. No obstante lo anterior, los años 2013 y 2014 se iniciaron solo 54 procedimientos sancionatorios originados por este motivo. De esta forma, las estadísticas de denuncias deben servir de base para establecer nuevas formas y mecanismos tanto de fiscalización como de sanción, que permitan aumentar la cobertura y la capacidad de procesar dichos trámites. En ese mismo sentido, a la fecha la institución solo cuenta con oficinas en Antofagasta, Santiago y Valdivia, siendo necesario ampliar la cobertura, a fin de que la ciudadanía pueda tener mayores facilidades para ejercer sus derechos.

\section{Tareas pendientes para una mejor fiscalización, sanción y cumplimiento ambiental}

El funcionamiento de la nueva institucionalidad ambiental en Chile ha tenido un punto de inflexión con la implementación y puesta en marcha de la Superintendencia del Medio Ambiente. En ese contexto, resultan evidentes los avances en materia de fiscalización y cumplimiento ambiental respecto del modelo de coordinación desarrollado e implementado desde 1994 a 2012.

No obstante lo anterior, existen aspectos legislativos, administrativos y prácticos que deben ser revisados y modificados, en caso de que sea pertinente, para que la nueva institucionalidad ambiental pueda ser efectiva y eficiente. 
Una de las recomendaciones de la OCDE y CEPAL (2005) indicaba la necesidad de incrementar competencias en el ámbito regional, esto con el objeto de limitar los conflictos de interés. En la práctica, las competencias a nivel regional se encuentran limitadas, debido a que la legislación tiene una fuerte impronta centralista, y si bien se están creando oficinas regionales, estas son supervisadas y administradas desde el nivel central, y no de forma descentralizada desde un Gobierno Regional. En el mismo sentido, Guiloff (2010), en forma previa a la promulgación de la Ley 20.417, indicaba "donde existe mayor consenso es en la necesidad de que el organismo fiscalizador tenga una cierta autonomía frente a la autoridad central” (p. 46). En este sentido, es necesario avanzar en la implementación de un modelo descentralizado y efectivamente independiente de la autoridad central. Una oportunidad para esto lo constituye la Agenda de Descentralización, cuyas conclusiones, provenientes de la Comisión Asesora Presidencial en Descentralización y Desarrollo Regional, fueron entregadas el 7 de octubre de 2014 a la presidenta Michelle Bachelet (Gobierno de Chile, 2014). De acuerdo con la citada Comisión, existen diez medidas esenciales, entre las cuales se encuentra la referida al fortalecimiento de la institucionalidad pública de las regiones, donde se plantea la transferencia de competencias desde el nivel central hacia los niveles regionales y municipales como una forma de asegurar la descentralización de las decisiones. En ese mismo contexto, entonces, el traspaso de competencias debe considerar la fiscalización y sanción ambiental, lo que permitiría superar los desbalances territoriales mostrados en términos de fiscalización o las situaciones de Justicia Ambiental, asociados a la interpretación de los Planes de Descontaminación en distintas áreas urbanas del país.

Adicionalmente, en el mismo sentido anterior -es decir, para avanzar en mayor autonomía respecto del nivel central, de forma de establecer una independencia del organismo medioambiental respecto de los gobiernos de turno-, se debe regular el nombramiento del superintendente del Medio Ambiente a fin de que sea realizado por el Congreso de la República, de forma similar a como se formaliza el de contralor general de la República o del fiscal nacional económico. Hoy en día, la alta rotación de profesionales en el aparato medioambiental asociada a los cambios de gobierno aparece como un importante problema. Según De la Puente (2011), los países desarrollados plantean que el sistema de empleo público que permite afrontar los nuevos desafíos es aquel que hace posible atraer los profesionales que la administración necesita, que estimula a los empleados para el cumplimiento eficiente de sus funciones y responsabilidades, les proporciona la formación adecuada y les brinda suficientes oportunidades de promoción profesional, al tiempo que facilita una gestión racional y objetiva, ágil y flexible del personal, atendiendo al continuo desarrollo de las nuevas tecnologías. En ese sentido, de acuerdo con Hansen y Lavanderos (2011) respecto de la gestión de personas en el Estado, un desafío es que "el sistema de Alta Dirección Pública funcione en su totalidad y eficientemente para ambos niveles jerárquicos, de tal modo de asegurar competencias relacionales suficientes en los directivos que asumen funciones en las reparticiones del Estado" (p. 93).

Asimismo, se debe implementar mejoras legales sustanciales para los mecanismos de incentivo al cumplimiento, sobre todo de Planes de Reparación, de forma de que no se solapen competencias entre los distintos organismos públicos, y sobre todo 
se limiten las funciones del Tribunal Ambiental a las que efectivamente por ley le corresponden. Además, es importante buscar fórmulas para mejorar o rediseñar el mecanismo de autodenuncias, de modo que permita cumplir el objetivo para el cual fue establecido, el cual fue incentivar el cumplimiento de los regulados.

Finalmente, es necesario destacar que continuar con las actividades de cooperación con organismos de otros países e instituciones internacionales, basada en un espíritu de colaboración y buena fe, permitirá aprender de la experiencia de otros colegas e instituciones y compartir prácticas y procedimientos de fiscalización (Pérez, Monckeberg \& Bergamini, 2014). Lo anterior basado en la premisa fundamental de maximizar los objetivos de cumplimiento con la legislación ambiental en vigor en el país (Monckeberg, Bergamini \& Pérez, 2015).

\section{Referencias bibliográficas}

Biblioteca del Congreso Nacional (вСN). (2010). Historia de la Ley $N^{\circ} 20.417$ Crea el Ministerio, el Servicio de Evaluación Ambiental y la Superintendencia de Medio Ambiente. Biblioteca del Congreso Nacional. Disponible en http://www.leychile.cl/Navegar/scripts/obtiene archivo?id=recursoslegales/10221.3/3929/6/HL20417.pdf

Cordero, L. (1996). Institucionalidad ambiental en el Derecho chileno. Santiago: Editorial Jurídica ConoSur.

De la Puente, R. (2011). Empleo público y derechos laborales. En J. Ensignia \& A. Führer (Eds.), Reforma del Estado y relaciones laborales en el Chile de hoy (pp. 12-22). Santiago: Friedrich-Ebert-Stiftung. Disponible en http://library.fes.de/pdf-files/bueros/ chile/08480.pdf

Gobierno de Chile, Comisión Asesora Presidencial en Descentralización y Desarrollo Regional. (2014). Propuesta de Politica de Estado y Agenda para la Descentralización y el Desarrollo Territorial de Chile. Hacia un pais desarrollado y justo [En línea]. Disponible en http:// biblio.red-dete.org/wp-content/uploads/2014/11/PROPUESTAS-COMISIONDESCENTRALIZACION-FINAL.pdf

Guiloff, M. (2010). Reforma a la institucionalidad ambiental: un análisis desde el diseño institucional. En D. Hervé, M. Guiloff \& R.L. Pérez (Coords.), Reforma a la institucionalidad ambiental, antecedentes y fundamentos (pp. 27-55). Santiago: Ediciones Universidad Diego Portales.

Hansen, C. \& Lavanderos, R. (2011). Política de desarrollo de las personas en el sector público: balance y proyecciones. En J. Ensignia \& A. Führer (Eds.), Reforma del Estado y relaciones laborales en el Chile de hoy (pp. 72-93). Santiago: Friedrich-Ebert-Stiftung. Disponible en http://library.fes.de/pdf-files/bueros/chile/08480.pdf

Hervé, D. (2010). Noción y elementos de la justicia ambiental: directrices para su aplicación en la planificación territorial y en la evaluación ambiental estratégica. Revista de Derecho (Valdivia), 23(1), 9-36. http://dx.doi.org/10.4067/S0718-09502010000100001

Instituto Nacional de Estadísticas (INE). (2015). Proyecciones de Población 2014, [En línea]. Disponible en http://www.ine.cl/canales/chile_estadistico/demografia_y_vitales/ proyecciones2014/proyecciones-de-poblacion-2014.xlsx 
Ministerio del Medio Ambiente (MMA). (2014). Planes de Descontaminación Atmosférica, Estrategia 2014-2018 [En línea]. Disponible en http://www.mma.gob.cl/1304/w3article-56174.html

Monckeberg, J.C., Bergamini, K. \& Pérez, C. (2015). Environmental enforcement networking efforts in Chile: Lessons learnt and challenges ahead. En M. Faure, P. De Smedt \& A. Stas, Environmental enforcement networks; concepts, implementation and effectiveness (pp. 470-481). Londres: Edward Elgar Publishing. doi: 10.4337/9781783477401.00037

Olivares, A. (2010). El nuevo marco institucional ambiental en Chile. Revista Catalana de Dret Ambiental, 1(1), 1-23. Disponible en http://www.rcda.cat/index.php/rcda/article/ view/33

Organización para la Cooperación y el Desarrollo Económicos (OCDE) \& Comisión Económica para América Latina y el Caribe (CEPAL). (2005). Evaluaciones del desempeño ambiental, Chile. CEPAL, Santiago, Chile. Disponible en http://www.sinia.cl/1302/articles-57009_ EDA2005_ocDE_CEPAL.pdf

Pastén, B. \& Parot, G. (2012). Diversificación de la reparación del daño ambiental en Chile: perspectivas para los Planes de Reparación. Justicia Ambiental (Revista de Derecho Ambiental), 4 (dic. 28).

Pérez, C., Monckeberg, J.C. \& Bergamini, K. (2014). Creation of the South American Network for Environmental Enforcement and Compliance: International Cooperative Efforts for a Better Environment. En Proceedings 34th Annual Conference of the International Association for Impact Assessment. IAIA14 Impact Assessment for Social and Economic Development, April 8-11 2014, Viña del Mar, Chile.

Segundo Tribunal Ambiental. (2015). Causa D-1-2013 Estado de Chile/Centro de Residuos Orgánicos Colhue S.A. [En línea]. Disponible en http://goo.gl/k7xixv

Superintendencia del Medio Ambiente (SMA). (2014a). Resolución Exenta 4/2014 Instruye y fija programa y subprogramas sectoriales de fiscalización ambiental de resoluciones de calificación ambiental para el año 2014, [En línea]. Disponible en http://goo.gl/ UMNBRg

Superintendencia del Medio Ambiente (sMA). (2014b). Resolución Exenta 769/2014 Fija Programa y Subprogramas de Fiscalización Ambiental de Resoluciones de Calificación Ambiental para el año 2015, [En línea]. Disponible en http://goo.gl/7Pv5cU

Superintendencia del Medio Ambiente (sma). (2014c). Cuenta Pública. Programa y Subprograma de Fiscalización 2013. Documento elaborado por J.C. Monckeberg, Superintendente del Medio Ambiente Santiago: sma. Disponible en http://pac.sma.gob.cl/sites/default/ files/Cuenta\%20Publica\%20_0.pdf

Superintendencia del Medio Ambiente (sma). (2015). Presentación Cuenta Pública sma 2014 [En línea]. Disponible en http://goo.gl/ezbJW9 